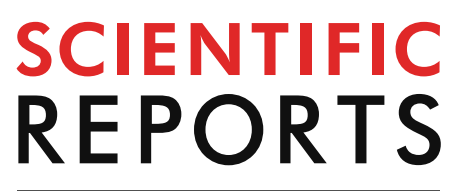

natureresearch

Check for updates

\title{
The prognostic significance of VISTA and CD33-positive myeloid cells in cutaneous melanoma and their relationship with PD-1 expression
}

\author{
Jae Won Choi ${ }^{1,2}$, Young Jae Kim ${ }^{1,2}$, Kyung A. Yun ${ }^{1}$, Chong Hyun Won ${ }^{1}$, Mi Woo Lee ${ }^{1}$, \\ Jee Ho Choi ${ }^{1}$, Sung Eun Chang ${ }^{1,3 凶}$ \& Woo Jin Lee ${ }^{1,3 凶}$
}

V-domain Ig suppressor of T-cell activation (VISTA), which mediates immune evasion in cancer, is mainly expressed on hematopoietic cells and myeloid cells in the tumor. We evaluated correlations among the expression of VISTA, the myeloid-derived suppressor cell marker CD33, and programmed death-1 (PD-1), and determined their relationships with clinicopathological characteristics and disease outcomes in melanoma. Diagnostic tissue from 136 cases of melanoma was evaluated by immunohistochemistry for CD33, VISTA, and PD-1 expression. Dual immunofluorescence using CD33 and VISTA antibodies was performed. VISTA expression positively correlated with CD33 expression in melanoma tissue. Dual immunofluorescence staining revealed that VISTA was expressed by CD33positive myeloid cells. PD-1 expression correlated with CD33 and VISTA expression. CD33 and VISTA expression were significantly associated with negative prognostic factors, including a deeper Breslow thickness and an advanced stage of disease. High expression of either CD33 or VISTA was associated with worse survival. Positivity for both VISTA and PD-1 predicted worse survival. Multivariate analysis showed that both CD33 and VISTA expression were independent prognostic factors in cutaneous melanoma. VISTA and CD33 expression are independent unfavourable prognostic factors in melanoma, which suggests their potential as therapeutic targets.

Myeloid-derived suppressor cells (MDSCs) are bone marrow-derived myeloid progenitors that include macrophages, granulocytes, dendritic cells, and immature myeloid cells ${ }^{1,2}$. MDSCs play important roles in disease progression and immune evasion in many types of cancer $^{3-5}$. There are two subpopulations of MDSCs, monocytic MDSCs and granulocytic MDSCs ${ }^{6,7}$. MDSCs typically express the common myeloid markers CD33 and CD11 ${ }^{8}$.

$\mathrm{V}$-domain Ig suppressor of T-cell activation (VISTA) is a recently identified negative regulator of T-cellmediated immune responses in cancer ${ }^{9-12}$. In contrast to programmed cell death protein-1 (PD-1), which is primarily found on activated and exhausted T cells, VISTA is mainly expressed on hematopoietic cells and myeloid cells including neutrophils, monocytes, macrophages, and dendritic cells ${ }^{13,14}$. Expression of VISTA is high in myeloid cells and low in CD4+ and CD8+ T cells and CD4+ FoxP3+ regulatory T cells ${ }^{9-11,15}$. As an immune checkpoint inhibitor with a different expression pattern than other previously identified checkpoints, VISTA could be a novel therapeutic marker in anticancer immunotherapy ${ }^{10}$. VISTA overexpression downregulates the immunity by suppression T-cell proliferation and production of T-cell cytokine such as IL-2 and IFN- $\gamma^{11}$. The inhibitory function of VISTA in anticancer immunity was demonstrated in mice transplanted with melanoma, in which blocking of VISTA induced antitumor immunity by increasing tumor-specific CD4+ and CD8+ T cells and decreasing FoxP3+ regulatory T cells in the tumor microenvironment.10 Genetic deletion of VISTA (Vsir) resulted in increased production of inflammatory cytokines and chemokines in a mice model ${ }^{16}$.

${ }^{1}$ Department of Dermatology, Asan Medical Center, University of Ulsan College of Medicine, 88, Olympic-ro 43-gil, Songpa-gu, Seoul 05505, Korea. ${ }^{2}$ These authors contributed equally: Jae Won Choi and Young Jae Kim. ${ }^{3}$ These authors jointly supervised this work: Sung Eun Chang and Woo Jin Lee. ${ }^{\varpi}$ email: csesnumd@gmail.com; vucm79@gmail.com 
Recent study noticed the role of MDSC-mediated immunosuppression in tumor progression and found that MDSCs can contribute to patient resistance to immune checkpoint inhibition ${ }^{17}$. It was also reported that reversed MDSC-mediated suppression with myeloid cell receptor tyrosine kinases inhibition consequences the augmentation of anti-PD-1 therapy in melanoma ${ }^{18}$.

These findings imply close underlying correlation between MDSCs and PD-1 in tumor microenvironment. Likewise, the expression of VISTA positively correlated with the expression of PD-ligand 1 (PD-L1) and PD-1, and the numbers of CD8+ T cells and CD68+ macrophages, in the tumor microenvironment in non-small cell lung cancer ${ }^{19}$. In oral squamous cell carcinoma, VISTA expression correlated with MDSC markers (CD11b and CD33) and was associated with increased expression of IL13Ra2, an important cytokine involved in tumor metastasis and recruitment of MDSCs ${ }^{20}$.

Although the prognostic significance of VISTA and its correlation with PD-1 expression has been evaluated in cutaneous melanoma ${ }^{21}$, the association between VISTA and CD33+ MDSCs has not been elucidated. Recently, combinations of immune checkpoint inhibitors have emerged as anticancer therapies. For example, CA-170, is an oral inhibitor which targets both PD-L1 and VISTA revealed remarkable anti-tumor effects in preclinical study and the phase I trial in patients with advanced solid tumors including melanoma is currently investigated (NCT02812875) ${ }^{22}$. In this aspect, evaluation of the relationship between PD-1 and VISTA or CD33 expression is important. However, many questions are still remained for VISTA and its interaction with other immune check point molecules.

In the present study, we examined the expression of VISTA in primary melanoma tissue and analyzed its association with clinicopathological features and clinical outcome. In addition, the correlation between the expression of VISTA and MDSC markers was evaluated. We also analyzed the association between CD33/VISTA and PD-1 to evaluate the potential for combination therapy with MDSC-targeting agents and anti-PD-1 antibodies.

\section{Materials and methods}

This study was approved by the Institutional Review Board of Asan Medical Center. A database at Asan Medical Center was searched for cases of cutaneous melanoma that were confirmed by skin biopsy between January 2001 and December 2016. All experiments were performed in accordance with relevant guidelines and regulations. Informed consent to publish identifying informations was obtained from study participants.

Evaluation of VISTA and CD33 expression in cutaneous melanoma. Paraffin-embedded sections were immunostained with anti-VISTA (1:200, ProSci Incorporation, CA, USA), anti-CD33 (1:100, Leica Biosystems, Newcastle, UK), or anti-PD-1 (1:100, Ventana, Tucson, AZ, USA) antibodies. All slides were evaluated and scored under light microscopy, analyzed and photographed by using Olympus cellSens software (version 1.4). Tumor-infiltrating inflammatory cells (TIICs) were identified based on morphology in hematoxylin-eosin (H\&E)-stained sections. Immunohistochemical staining was measured by semiquantitative assessment of positive staining in TIICs. For VISTA, CD33, and PD-1 antibodies, the percentage of TIICs showing positive membrane staining was determined. The intensity of staining was determined on a scale of $0-3(0=<5 \%, 1=5-20 \%$, $2=20-50 \%$, and $3=>50 \%$ of TIICs). Cases with a score $\geq 1$ were considered positive. Scores of 2 or 3 were considered to represent high expression of VISTA, CD33, and PD-1. The numbers of VISTA-, PD-1-, and CD33positive TIICs were counted in five representative high-powered fields (200X magnification) and averaged. All melanoma tissues evaluated in the study are the primary tumor tissues from the primary cutaneous melanoma tissues. All samples were evaluated independently by two investigators (LWJ, CJW).

Immunofluorescence staining. Dual immunofluorescence (IF) staining was carried out using formalin-fixed, paraffin-embedded sections after heat antigen retrieval following standard protocols. The following primary antibodies were used: anti-VISTA (1:50, ProSci Incorporation, Poway, CA, USA), anti-CD33 (1:50, Leica Biosystems, Newcastle, UK), and anti-PD-1 (1:50, R\&D Systems, Minneapolis, MN, USA). The secondary antibodies and dilutions used for IF staining were as follows: goat anti-rabbit IgG(H+L)-FITC (Southern Biotech, Birmingham, AL, USA; cat. no. 4055-02; 1:500), rabbit anti-goat IgG(H+L)-FITC (Southern Biotech; cat. no. 6160-02, 1:500), and cross-adsorbed goat anti-mouse IgG(H+L)-AlexaFluor 546 (ThermoFisher Scientific, Waltham, MA, USA; cat. no. A-11003; 1:500).

Variables of interest. The clinical features of the patient and the primary lesion, including age at diagnosis, sex, the location of the lesion, lymph node (LN) invasion, and distant visceral metastasis, were determined through the medical records and preoperative photographs. Overall survival (OS) was calculated from the date of the initial diagnosis to either the date of death from any cause or the date of the last follow-up examination. Progression-free survival (PFS) was calculated from the date of the initial diagnosis to the first day of disease progression or recurrence, or the last follow-up. Survival curves were calculated from medical records. The stage of disease was determined according to the 8th edition of the American Joint Committee on Cancer (AJCC) ${ }^{23}$. Biopsy specimens were reviewed and the following data was collected: histopathologic subtype, Breslow thickness, ulceration, and vertical growth phase.

Statistical analysis. All analyses were performed using a statistical software package (SPSS, version 18.0; SPSS Inc., Chicago, IL). P-values of less than 0.05 were considered statistically significant. Survival was analyzed using the Kaplan-Meier method, and comparisons were made by log-rank testing. Prognostic factors independently associated with survival were identified by multivariate analysis using Cox proportional hazards regression modelling. Comparisons between subgroups were performed using a Chi-squared test or Fisher's exact test 


\begin{tabular}{|c|c|c|c|c|c|c|c|c|c|}
\hline & \multicolumn{3}{|c|}{ CD33 expression } & \multicolumn{3}{|c|}{ VISTA expression } & \multicolumn{3}{|c|}{ PD-1 expression } \\
\hline & $\begin{array}{l}\text { Score } 0 \text { or } 1 \\
(n=93)\end{array}$ & $\begin{array}{l}\text { Score } 2 \text { or } 3 \\
(n=43)\end{array}$ & $P$ value & $\begin{array}{l}\text { Score } 0 \text { or } 1 \\
(n=99)\end{array}$ & $\begin{array}{l}\text { Score } 2 \text { or } 3 \\
(n=37)\end{array}$ & $P$-value & $\begin{array}{l}\text { Score } 0 \text { or } 1 \\
(\mathrm{n}=69)\end{array}$ & \begin{tabular}{|l|} 
Score 2 or 3 \\
$(n=67)$
\end{tabular} & $P$ value \\
\hline $\begin{array}{l}\text { Breslow } \\
\text { thickness, } \\
\text { mm }\end{array}$ & & & $0.009^{*}$ & & & $0.002^{*}$ & & & 0.088 \\
\hline $\begin{array}{l}\leq 1(\mathrm{~T} 1) \\
(n=24)\end{array}$ & 20/93 (21.5) & $4 / 43(9.3)$ & & 20/99 (20.2) & $4 / 37(10.8)$ & & $15 / 69(21.7)$ & $9 / 67(13.4)$ & \\
\hline $\begin{array}{l}>1 \text { to } \leq 2 \\
(\mathrm{~T} 2)(n=49)\end{array}$ & $37 / 93(39.8)$ & $12 / 43(27.9)$ & & $41 / 99(41.4)$ & $8 / 37(21.6)$ & & $27 / 69(39.1)$ & $22 / 67(32.8)$ & \\
\hline $\begin{array}{l}>2 \text { to } \leq 4 \\
\text { (T3) }(n=35)\end{array}$ & $25 / 93$ (26.9) & $10 / 43(23.3)$ & & 25/99 (25.3) & $10 / 37(27.0)$ & & $19 / 69(27.5)$ & $16 / 67(23.9)$ & \\
\hline $\begin{array}{l}>4(\mathrm{~T} 4) \\
(n=28)\end{array}$ & $11 / 93(11.8)$ & $17 / 43(39.5)$ & & $13 / 99(13.1)$ & $15 / 37(40.5)$ & & $8 / 69$ (11.6) & 20/67 (29.9) & \\
\hline Ulceration & & & $0.040^{*}$ & & & $0.015^{*}$ & & & 0.210 \\
\hline Yes $(n=38)$ & 21/93 (22.6) & $17 / 43(39.5)$ & & $22 / 99(22.2)$ & & & $16 / 69(23.2)$ & $22 / 67(32.8)$ & \\
\hline No $(n=98)$ & 72/93 (77.4) & $26 / 43(60.5)$ & & 77/99 (77.8) & $16 / 37(43.2)$ & & $53 / 69(76.8)$ & $45 / 67(67.2)$ & \\
\hline $\begin{array}{l}\text { Vertical } \\
\text { growth phase }\end{array}$ & & & 0.063 & & $21 / 37(56.8)$ & $0.041^{*}$ & & & 0.452 \\
\hline Yes $(n=51)$ & $30 / 93(32.3)$ & $21 / 43(48.8)$ & & $32 / 99(32.3)$ & $19 / 37(51.4)$ & & $28 / 69(40.6)$ & $23 / 67(34.3)$ & \\
\hline No $(n=85)$ & 63/93 (67.7) & $22 / 43(51.2)$ & & 67/99 (67.7) & $18 / 37(48.6)$ & & $41 / 69(59.4)$ & $44 / 67(65.7)$ & \\
\hline \multicolumn{10}{|l|}{ Sex } \\
\hline Male $(n=74)$ & $46 / 93$ (49.5) & $28 / 43(65.1)$ & 0.088 & $55 / 99(55.6)$ & 19/37 (51.4) & 0.661 & $39 / 69(56.5)$ & $35 / 67(52.7)$ & 0.616 \\
\hline $\begin{array}{l}\text { Female } \\
(n=62)\end{array}$ & $47 / 93(50.5)$ & $15 / 43(34.9)$ & & $44 / 99(44.4)$ & $18 / 37(48.6)$ & & $30 / 69(43.5)$ & $32 / 67(47.8)$ & \\
\hline \multicolumn{10}{|l|}{ Age } \\
\hline $\begin{array}{l}<60 \text { years } \\
(n=74)\end{array}$ & $54 / 93(58.1)$ & $20 / 43(46.5)$ & 0.208 & $56 / 99(56.6)$ & $18 / 37(48.6)$ & 0.409 & $36 / 69(52.2)$ & $38 / 67(56.7)$ & 0.595 \\
\hline $\begin{array}{l}\geq 60 \text { years } \\
(n=62)\end{array}$ & $39 / 93$ (41.9) & $23 / 43(53.5)$ & & 43/99 (43.4) & $19 / 37(51.4)$ & & $33 / 69(47.8)$ & $29 / 67(43.3)$ & \\
\hline \multicolumn{10}{|c|}{ Extracutaneous involvement } \\
\hline $\begin{array}{l}\text { Lymph node } \\
(n=28)\end{array}$ & $12 / 93(12.9)$ & $16 / 43(37.2)$ & $0.001^{*}$ & $13 / 99(13.1)$ & $15 / 37(40.5)$ & $0.000^{*}$ & $17 / 69(24.6)$ & $11 / 67(16.4)$ & 0.236 \\
\hline $\begin{array}{l}\text { Viscera } \\
(n=12)\end{array}$ & $5 / 93(5.4)$ & $7 / 43(16.3)$ & 0.051 & $6 / 99(6.1)$ & & 0.063 & $6 / 69(8.7)$ & $6 / 67(9.0)$ & 0.957 \\
\hline AJCC stage & & & $0.005^{*}$ & & $6 / 37(16.2)$ & $0.008^{*}$ & & & 0.192 \\
\hline $\mathrm{I} / \mathrm{II}(n=103)$ & $77 / 93(82.8)$ & $26 / 43(60.5)$ & & $81 / 99(81.8)$ & $22 / 37(59.5)$ & & $49 / 69(71.0)$ & $54 / 67(80.6)$ & \\
\hline $\mathrm{III} / \mathrm{IV}(n=33)$ & $16 / 93(17.2)$ & $17 / 43(39.5)$ & & $18 / 99(18.2)$ & $15 / 37(40.5)$ & & $20 / 69(29.0)$ & $13 / 67(19.4)$ & \\
\hline
\end{tabular}

Table 1. Clinico-histopathological characteristics of the 136 cutaneous melanomas according to CD33, VISTA and PD-1 expression. ${ }^{*}$ Statistically significant. VISTA, V-domain Ig suppressor of T-cell activation; AJCC, American Joint Committee on Cancer.

for categorical variables, and a t-test or the Mann-Whitney test for continuous variables. Pearson's correlation coefficient was used to evaluate associations among continuous variables.

\section{Results}

A total of 136 cases of cutaneous melanoma were included in the study. The demographic data and clinical features of the patients are summarized in Table 1.

Expression of CD33 and VISTA and their association with clinicopathological features. Of 136 patients, CD33 (Fig. 1A,C) and VISTA (Fig. 1B,D) were highly expressed in $43(31.6 \%)$ and 37 (27.2\%) patients, respectively. Clinicopathological variables were stratified by the expression of these proteins in the tumor to identify correlations (Table 1).

High expression of $\mathrm{CD} 33$ was associated with clinicopathological variables that have negative prognostic indexes. There were significant correlations between high expression of CD33 and pathological variables such as a deeper Breslow thickness $(P=0.009)$ and the presence of ulceration $(P=0.040)$. High CD33 expression was also associated with a higher likelihood of LN involvement $(P=0.001)$ and an advanced AJCC stage $(P=0.005$; Table 1).

High VISTA expression was also associated with poor clinicopathological features. There was a significant association between high expression of VISTA and pathological features such as a deeper Breslow thickness $(P=0.002)$, the presence of ulceration $(P=0.015)$ and higher frequency of vertical growth phase $(P=0.041)$. More tendency of $\mathrm{LN}$ involvement $(P<0.001)$ and advanced AJCC stage $(P=0.008)$ (Table 1$)$ were also associated with high expression of VISTA. 


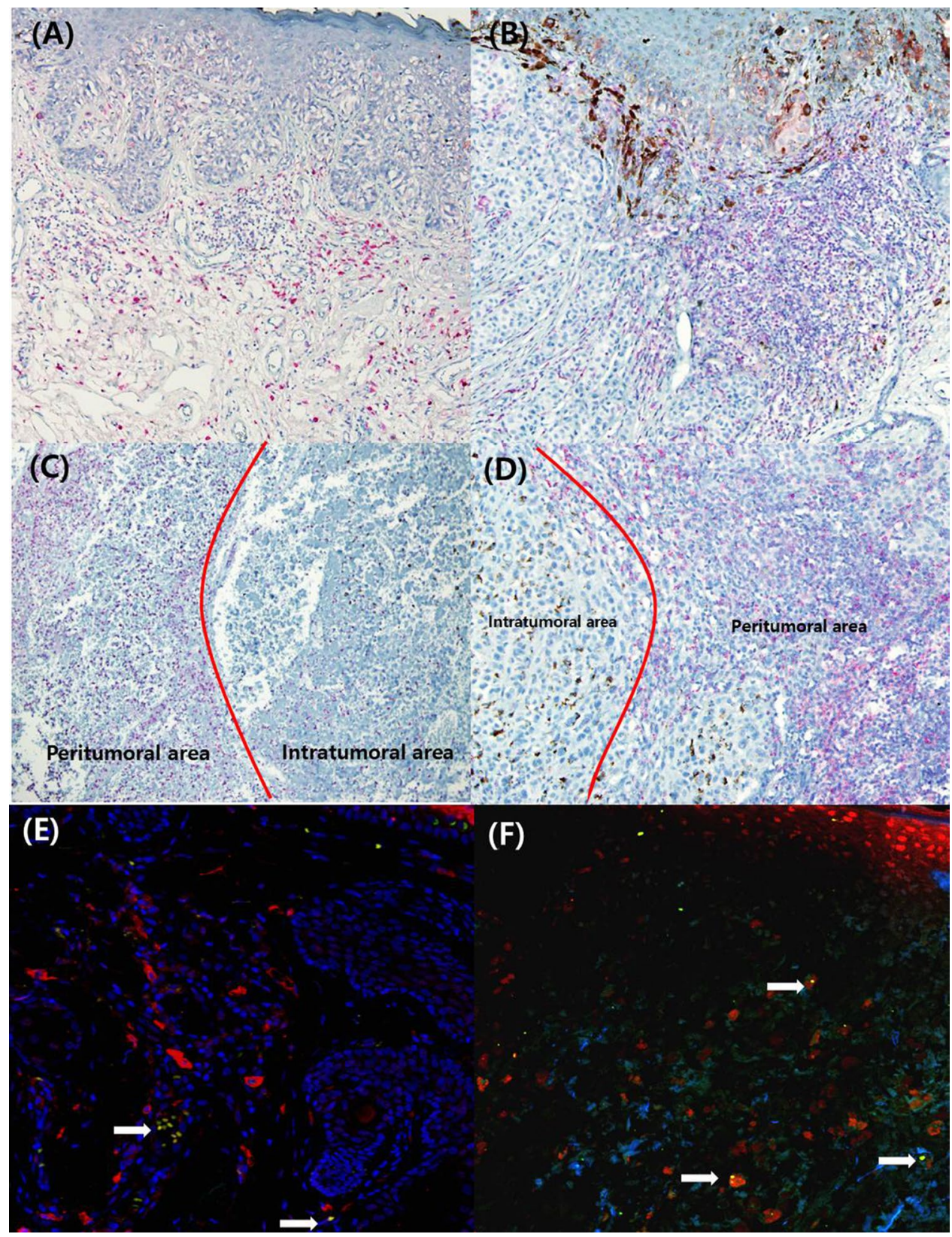

Figure 1. Expression of CD33 and VISTA in cutaneous melanoma. (A) High intratumoral expression (immunohistochemical score of 3, red color, cytoplasmic) of CD33 in acral melanoma $(\times 100)$. (B) High intratumoral expression (score 3, red color, cytoplasmic) of VISTA in nodular melanoma $(\times 100)$. (C) High peritumoral expression (score 3, red color, cytoplasmic) of CD33 in acral melanoma $(\times 100)$. (D) High peritumoral expression (score 3, red color, cytoplasmic) of VISTA in nodular melanoma $(\times 200)$. (E) Dual immunofluorescence staining of (A) CD33 and VISTA (CD33, red, cytoplasmic; VISTA, green, cytoplasmic) and (F) PD-1 and VISTA (PD-1, red, cytoplasmic; VISTA, green, cytoplasmic). Double-positive cells are shown in yellow (white arrow). VISTA, V-domain Ig suppressor of T-cell activation; PD-1, programmed cell death protein-1.

Of 136 patients, 67 (49.3\%) showed high PD-1 expression (immunohistochemical score of 2 or 3 ). However, high PD-1 expression was not associated with clinicopathological variables that have negative prognostic indexes, such as a deeper Breslow thickness $(P=0.088)$, the presence of ulceration $(P=0.210)$ and higher frequency of vertical growth phase $(P=0.452)$. Among these 67 patients, $27(40.3 \%)$ and $24(35.8 \%)$ showed high expression of CD33 and VISTA, respectively (Table 2). There was a significant correlation between PD-1 expression and expression of CD33 $(P=0.032)$ and VISTA $(P=0.026)$. 


\begin{tabular}{|c|c|c|c|c|c|c|}
\hline & \multicolumn{2}{|c|}{ VISTA expression, $n$ (\%) } & \multirow[b]{2}{*}{$P$ value } & \multicolumn{2}{|c|}{ CD33 expression, n (\%) } & \multirow[b]{2}{*}{$P$ value } \\
\hline & Score 0 or $1(n=99)$ & Score 2 or $3(n=37)$ & & Score 0 or $1(n=93)$ & Score 2 or $3(n=43)$ & \\
\hline CD33 expression & & & $<0.001^{*}$ & & & \\
\hline Score 0 or $1(n=93)$ & 88/99 (88.9) & $5 / 37(13.5)$ & & NA & & \\
\hline Score 2 or $3(n=43)$ & $11 / 99(11.1)$ & $32 / 37(86.5)$ & & & & \\
\hline \begin{tabular}{|l|} 
PD-1 expression \\
\end{tabular} & & & $0.026^{*}$ & & & $0.032^{*}$ \\
\hline Score 0 or $1(n=69)$ & $56 / 99(56.6)$ & $13 / 37(35.1)$ & & $53 / 93(57.0)$ & $16 / 43(37.2)$ & \\
\hline Score 2 or $3(n=67)$ & 43/99 (43.4) & $24 / 37(64.9)$ & & $40 / 93(43.0)$ & $27 / 43(62.8)$ & \\
\hline
\end{tabular}

Table 2. Correlations among CD33, VISTA, and PD-1 expression. ${ }^{\star}$ Statistically significant. VISTA, V-domain Ig suppressor of T-cell activation; PD-1, programmed cell death protein-1.

Expression of VISTA on CD33-positive MDSCs. Double IF staining of melanoma tissue showed that CD33-positive cells also expressed VISTA (Fig. 1E). Of 43 cases with high CD33 expression, 32 (74.4\%) also showed high expression of VISTA. There was a significant correlation between the expression of CD33 and the expression of VISTA (Table 2, $P<0.001$ ). Double IF staining showed that PD-1-positive cells also expressed VISTA (Fig. 1F).

Prognostic significance of CD33 and VISTA expression. When all patients were combined into a single cohort, the 5 -year OS rate was 52\%, and the median OS was 62.0 months [ $95 \%$ confidence interval (CI) 50.12-79.52 months].

Median OS was significantly shorter in patients with high expression of CD33 (56.0 months; $95 \%$ CI 38.51-73.49 months) compared with those with low expression of CD33 (81.0 months; $95 \%$ CI 64.64-97.35 months) $(P=0.004$, Fig. 2A). PFS was also significantly better in patients with low expression of CD33 $(P<0.001)$. Patients with high expression of VISTA (58.0 months; 95\% CI 30.55-85.47 months) had inferior median OS compared with patients with low expression of VISTA (79.0 months; $95 \%$ CI $62.00-95.42$ months) $(P=0.017$, Fig. 2B). PFS was also affected by the expression of VISTA $(P=0.008)$. High expression of both CD33 and VISTA predicted inferior median OS (CD33 high VISTA ${ }^{\text {high }}$ : 61.0 months, $95 \%$ CI 35.65-86.35 months; and non-CD33 ${ }^{\text {high }}$ VISTA ${ }^{\text {high }}$ : 79.0 months, 95\% CI 63.09-94.87 months) $(P=0.032$, Fig. 2C) and PFS $(P=0.004)$. Patients with low expression of both CD33 and VISTA (CD33 ${ }^{\text {low } V I S T A ~}{ }^{\text {low: }} 81.0$ months, 95\% CI 61.39-199.60 months) showed significantly better median OS than patients with high expression of either CD33 or VISTA (Fig. 2D, $P=0.027$, CD $33^{\text {high }}$ VISTA $^{\text {low: }} 56.0$ months, $95 \%$ CI $28.28-83.72$ months; and CD33 ${ }^{\text {low}}$ VISTA ${ }^{\text {high }}$ : 65.0 months, 95\% CI not found). When survival was evaluated with respect to PD-1 expression, high expression of both VISTA and PD-1 was associated with worse median OS (VISTA ${ }^{\text {high }}$ PD- $1^{\text {high }}$ : 44.0 months, 95\% CI 30.4-57.59 months; and non-VISTA high PD- $1^{\text {high. }}$ : 71.0 months, 95\% CI 59.49-82.50 months) $(P=0.033)$. High expression of VISTA was also associated with OS in patients with high expression of PD-1 (VISTA high PD- $1^{\text {high: }}$ 44.0 months, 95\% CI 30.78-60.21 months; and VISTA ${ }^{\text {low }}$ PD- $1^{\text {high: }}: 81.0$ months, 95\% CI 31.82-111.17 months) ( $P=0.036$, Fig. 2E).

By univariate analysis, CD33 expression [hazard ratio (HR) 2.11; 95\% CI 1.21-4.23; $P=0.031$ ] and VISTA expression (HR 1.86; $95 \%$ CI 1.26-5.54; $P=0.039$ ) were associated with worse OS (Table 3). By multivariate analysis using these variables, CD33 expression (HR 1.61; 95\% CI 1.18-4.23; $P=0.041$ ) and VISTA expression (HR 1.51; 95\% CI 1.14-5.61; $P=0.046$ ) were independent prognostic markers for worse OS (Table 3).

\section{Discussion}

Immune checkpoints and suppressive immune cells such as MDSCs, regulatory T cells, and tumor-associated macrophages play important roles in tumor progression by enabling cancer cells to evade antitumor immunity 24,25 . MDSCs are a heterogeneous group of myeloid cells that suppress T-cell activity in the tumor microenvironment ${ }^{26}$. MDSCs can contribute to tumor invasion by inhibiting T-cell proliferation as well as by promoting tumor metastasis and angiogenesis ${ }^{26-30}$. Activated MDSCs in tumors contribute to the immunosuppressive microenvironment by producing arginine, reactive oxygen species, and immunosuppressive cytokines ${ }^{31,32}$. In addition, MDSCs express PD-L1 in response to tumor hypoxia and tumor-derived exosomes ${ }^{33,34}$. PD-1 expression in tumor-associated MDSCs is related to their proliferation and expression of molecules that inhibit the activity of antitumor T cells ${ }^{35}$. Therefore, blocking MDSCs could be a novel anticancer strategy that could enhance the antitumor effects of PD-1/PD-L1 inhibitors.

CD33 is a transmembrane receptor and myeloid differentiation antigen that is expressed on myeloid cells and is over-expressed in $90 \%$ of myeloid leukemias ${ }^{36}$. CD33 is found on maturing granulocytes, monocytes, and multipotent myeloid precursors, and is also expressed on subsets of activated $\mathrm{T}$ cells, natural killer cells, and $\mathrm{B}$ cells $^{37-39}$. Because of this expression pattern, CD33-directed therapies are used in malignancies such as chronic myelomonocytic leukemia, myelodysplastic syndrome, and acute lymphoblastic leukemia ${ }^{40-42}$.

The expression of CD33+ MDSCs in cutaneous melanoma and its relationship with PD-1 expression has not been evaluated. In this study, about 30\% of melanoma specimens showed high expression of CD33. Furthermore, high expression of CD33 was associated with poor clinicopathological variables and was an independent prognostic factor in melanoma. Moreover, CD33 expression correlated with PD-1 expression, which suggests the possibility that PD-1 expression could be affected by CD33-positive MDSCs in cutaneous melanoma. 
(A)

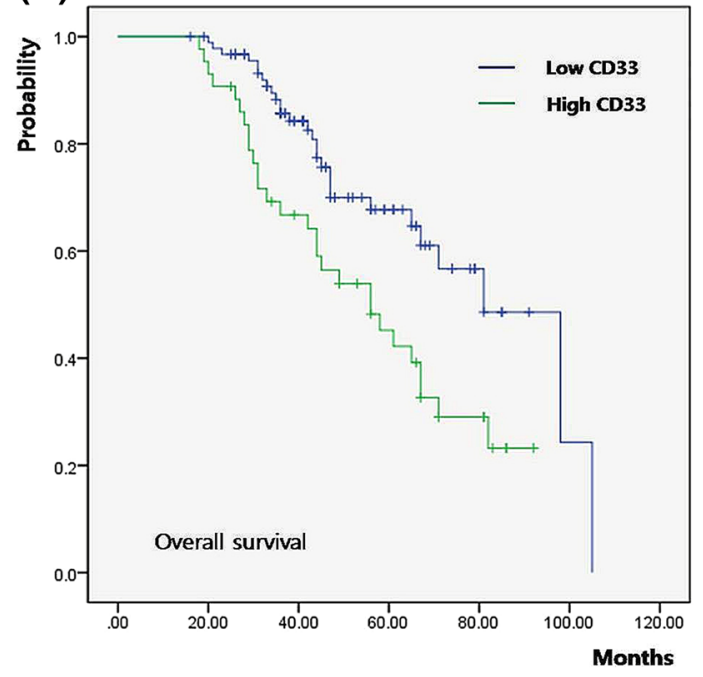

(C)

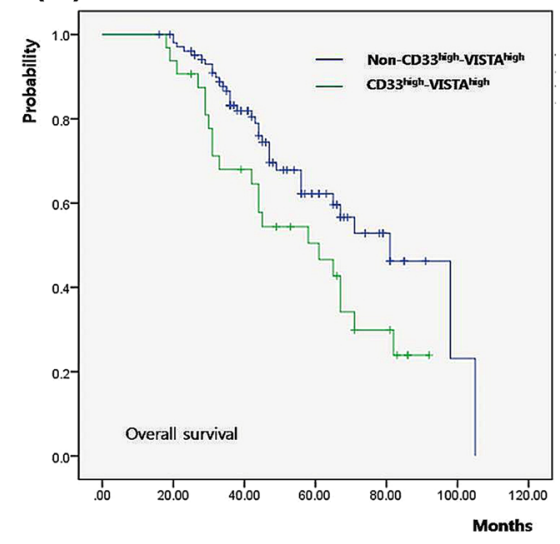

(D)

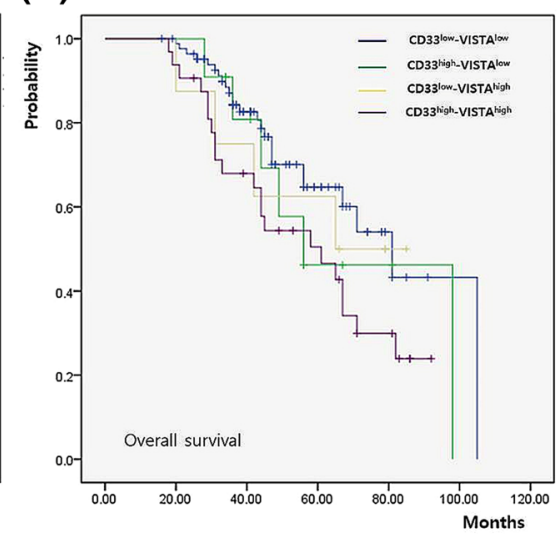

(B)

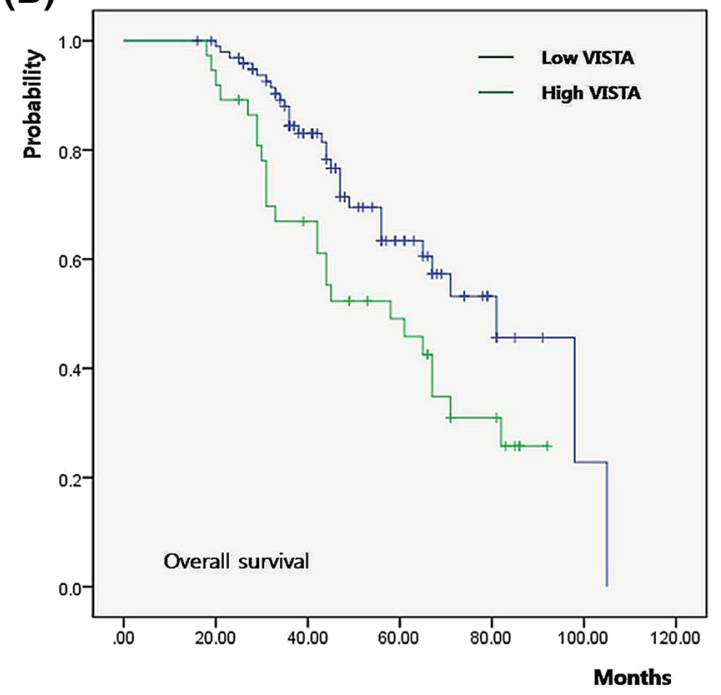

(E)

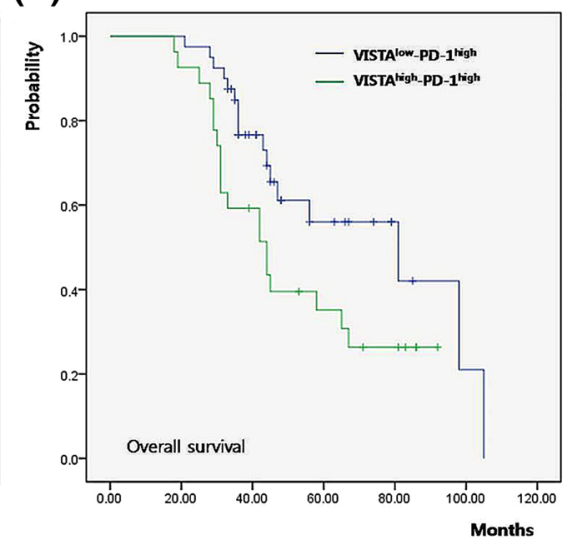

Figure 2. Patient survival according to CD33 and VISTA expression. (A) OS according to CD33 expression. (B) OS according to VISTA expression. (C) Worse OS in patients with high expression of both CD33 and VISTA. (D) OS according to the degree of CD33 and VISTA expression (E) Worse OS in cases with dual expression of VISTA and PD-1 among patients with high expression of PD-1. VISTA, V-domain Ig suppressor of T-cell activation; PD-1, programmed cell death protein-1.

As a negative immune checkpoint molecule with a different expression pattern than other previously described immune checkpoints, VISTA could be a novel therapeutic marker in anticancer immunotherapy ${ }^{10}$. VISTA overexpression downregulates the immunity by suppression T-cell proliferation and production of T-cell cytokine such as IL-2 and IFN- $\gamma^{11}$. The inhibitory function of VISTA in anticancer immunity was demonstrated in mice transplanted with melanoma, in which blocking of VISTA induced antitumor immunity by increasing tumorspecific CD4+ and CD8+ T cells and decreasing FoxP3+ regulatory T cells in the tumor microenvironment ${ }^{10}$. Genetic deletion of VISTA (Vsir) resulted in increased production of inflammatory cytokines and chemokines in a mice model ${ }^{16}$.

Consistent with previous data that VISTA is mainly expressed on hematopoietic cells and myeloid cells, and not exhausted or activated T cells, our study showed that VISTA expression correlated with the expression of the MDSC marker CD33 in cutaneous melanoma. Thirty-two of forty-three (74.4\%) patients with high expression of CD33 had high expression of VISTA. Double IF showed that CD33-positive MDSCs expressed VISTA. In a recent review of VISTA expression, high VISTA expression was found in myeloid cells, specifically microglia and neutrophils followed by monocytes, macrophages, and dendritic cells. Regarding T lymphocytes, VISTA is most highly expressed on naïve CD4+ and FoxP3+ regulatory T cells ${ }^{43}$.

VISTA blockade in the B16 melanoma model reduced the number of tumor-infiltrating monocytic MDSCs and increased the density of tumor-infiltrating effector T cells ${ }^{10}$. VISTA expression positively correlated with the expression of PD-1, PD-L1, and cytotoxic T-lymphocyte-associated antigen 4 (CTLA-4) in tumor-infiltrating immune cells, suggesting that these molecules simultaneously contribute to evasion of antitumor immunity and play a role in cancer progression ${ }^{20,21}$. We also found that VISTA expression was associated with clinicopathologic variables that are indexes of disease progression and poor survival, suggesting that VISTA is a prognostic biomarker and could be a target of antitumor immunotherapy. Furthermore, patients with high expression of both CD33 and VISTA had the worst survival in our cohort. Expression of VISTA on CD33-positive MDSCs could enhance their immunosuppressive function, resulting in tumor progression. In the present study, VISTA 


\begin{tabular}{|c|c|c|c|c|c|c|}
\hline \multirow[b]{2}{*}{ Covariate } & \multicolumn{3}{|l|}{ OS } & \multicolumn{3}{|l|}{ PFS } \\
\hline & HR & $95 \% \mathrm{CI}$ & $P$ value & HR & $95 \% \mathrm{CI}$ & $P$ value \\
\hline \multicolumn{7}{|l|}{ Univariate analysis } \\
\hline \multicolumn{7}{|l|}{ AJCC stage } \\
\hline Early versus advanced & 2.41 & $1.24-4.28$ & $0.023^{*}$ & 2.19 & $1.14-5.37$ & $0.045^{\star}$ \\
\hline \multicolumn{7}{|l|}{ Breslow thickness } \\
\hline $\mathrm{T} 1$ or $\mathrm{T} 2$ versus $\mathrm{T} 3$ or $\mathrm{T} 4$ & 2.19 & $1.21-5.55$ & $0.028^{*}$ & 1.98 & $1.29-6.11$ & $0.039^{*}$ \\
\hline \multicolumn{7}{|l|}{ CD33 expression } \\
\hline High versus low & 2.11 & $1.21-4.23$ & $0.031^{\star}$ & 1.98 & $1.16-4.55$ & $0.042^{*}$ \\
\hline \multicolumn{7}{|l|}{ VISTA expression } \\
\hline High versus low & 1.86 & $1.26-5.54$ & $0.039^{*}$ & 1.43 & $1.05-4.77$ & 0.053 \\
\hline \multicolumn{7}{|l|}{ PD-1 expression } \\
\hline High versus low & 0.745 & $0.31-3.29$ & 0.573 & 0.642 & $0.33-3.12$ & 0.528 \\
\hline \multicolumn{7}{|l|}{ Multivariate analysis } \\
\hline \multicolumn{7}{|l|}{ AJCC stage } \\
\hline Early versus advanced & 1.89 & $1.21-4.59$ & $0.042^{\star}$ & 1.87 & $1.11-4.21$ & 0.051 \\
\hline \multicolumn{7}{|l|}{ Breslow thickness } \\
\hline $\mathrm{T} 1$ or $\mathrm{T} 2$ versus $\mathrm{T} 3$ or $\mathrm{T} 4$ & 1.84 & $1.21-4.33$ & $0.044^{*}$ & 1.35 & $1.02-4.77$ & 0.064 \\
\hline \multicolumn{7}{|l|}{ CD33 expression } \\
\hline High versus low & 1.61 & $1.18-4.23$ & $0.041^{*}$ & 1.66 & $1.09-4.98$ & 0.056 \\
\hline \multicolumn{7}{|l|}{ VISTA expression } \\
\hline High versus low & 1.51 & $1.14-5.61$ & $0.046^{*}$ & 1.21 & $1.04-5.11$ & $0.040^{*}$ \\
\hline
\end{tabular}

Table 3. Univariate and multivariate analyses of OS and PFS. ${ }^{\star}$ Statistically significant. VISTA, V-domain Ig suppressor of T-cell activation; PD-1, programmed cell death protein-1; AJCC, American Joint Committee on Cancer; OS, Overall survival; PFS, progression-free survival; CI, confidence interval; HR, hazard ratio.

expression also correlated with PD-1 expression in melanoma tissue. The frequency of high expression was higher in PD-1 than VISTA in presenting study. We found that VISTA and PD-1 were both highly expressed in approximately $30 \%$ of melanoma cases. Among patients with high expression of PD-1, patients with high expression of VISTA (VISTA ${ }^{\text {high } P D-1 ~}{ }^{\text {high }}$ ) had a worse prognosis than patients with low expression of VISTA $\left(\right.$ VISTA $\left.^{\text {low }} \mathrm{PD}-1^{\text {high }}\right)$. These data suggest that VISTA expression can stratify survival in patients with high expression of PD-1. This finding also suggests that combination therapy with anti-VISTA and anti-PD-1 antibodies may be an effective approach for immunotherapy of cutaneous melanoma.

The combination of VISTA and PD-1 inhibitors synergistically enhanced antitumor immune responses in mouse models ${ }^{10,44}$. This suggests the therapeutic potential of targeting VISTA as an anticancer strategy. VISTA expression was previously reported to be an independent prognostic factor in melanoma ${ }^{21}$. Adaptive resistance to anti-PD-1 therapy also correlated with VISTA upregulation in melanoma patients, suggesting that VISTA blockade, possibly combined with PD-1 blockade, represents a potentially efficacious treatment strategy ${ }^{45}$. In addition, combination blockade of PD-1 and VISTA in a mice model did not result in overt autoimmunity, suggesting that this regimen could represent a less toxic alternative to anti-PD- 1 and anti-CTLA- 4 combination regimens ${ }^{44}$.

In conclusion, our study suggests that studies on the expression of CD33 and VISTA in melanoma may be of pivotal future interest. In light of the co-expression of PD-1 and VISTA, VISTA inhibitors may be of particular use in combination with immune checkpoint inhibitors such as PD-1 antibodies.

Received: 13 April 2020; Accepted: 10 August 2020

Published online: 01 September 2020

\section{References}

1. Monu, N. R. \& Frey, A. B. Myeloid-derived suppressor cells and anti-tumor T cells: a complex relationship. Immunol. Invest. 41, 595-613 (2012).

2. Haile, L. A., Greten, T. F. \& Korangy, F. Immune suppression: the hallmark of myeloid derived suppressor cells. Immunol. Invest. 41, 581-594 (2012).

3. Romano, A., Vetro, C. \& Adriani, M. Advances in understanding regulatory myeloid cells. Cancer Biol. Ther. 11, 923-926 (2011).

4. Brimnes, M. K. et al. Increased level of both CD4+FOXP3+ regulatory T cells and CD14+HLA-DR/low myeloid-derived suppressor cells and decreased level of dendritic cells in patients with multiple myeloma. Scand. J. Immunol. 72, 540-547 (2010).

5. Zhang, H. et al. Myeloid-derived suppressor cells inhibit T cell proliferation in human extranodal NK/T cell lymphoma: a novel prognostic indicator. Cancer Immunol. Immunother. 64, 1587-1599 (2015).

6. Sawant, A. \& Ponnazhagan, S. Myeloid-derived suppressor cells as osteoclast progenitors: a novel target for controlling osteolytic bone metastasis. Cancer Res. 73, 4606-4610 (2013).

7. Husain, Z., Huang, Y., Seth, P. \& Sukhatme, V. P. Tumor-derived lactate modifies antitumor immune response: effect on myeloidderived suppressor cells and NK cells. J. Immunol. 191, 1486-1495 (2013).

8. Qin, A. et al. Expansion of monocytic myeloid-derived suppressor cells dampens T cell function in HIV-1-seropositive individuals. J. Virol. 87, 1477-1490 (2013). 
9. Flies, D. B., Wang, S., Xu, H. \& Chen, L. Cutting edge: a monoclonal antibody specific for the programmed death-1 homolog prevents graft-versus-host disease in mouse models. J. Immunol. 187, 1537-1541 (2011).

10. Le Mercier, I. et al. VISTA regulates the development of protective antitumor immunity. Cancer Res. 74, 1933-1944 (2014).

11. Wang, L. et al. VISTA, a novel mouse Ig superfamily ligand that negatively regulates T cell responses. J. Exp. Med. 208, 577-592 (2011).

12. Flies, D. B. et al. Coinhibitory receptor PD-1H preferentially suppresses CD4 $4^{+} \mathrm{T}$ cell-mediated immunity. J. Clin. Invest. 124, 1966-1975 (2014).

13. Topalian, S. L., Drake, C. G. \& Pardoll, D. M. Immune checkpoint blockade: a common denominator approach to cancer therapy. Cancer Cell 27, 450-461 (2015).

14. Lines, J. L., Sempere, L. F., Broughton, T., Wang, L. \& Noelle, R. VISTA is a novel broad-spectrum negative checkpoint regulator for cancer immunotherapy. Cancer Immunol. Res. 2, 510-517 (2014).

15. Bharaj, P. et al. Characterization of programmed death-1 homologue-1 (PD-1H) expression and function in normal and HIV infected individuals. PLoS ONE 9, e109103 (2014).

16. Kloke, B. P. et al. Cancer immunotherapy achieves breakthrough status: 12 th annual meeting of the association for cancer immunotherapy (CIMT), Mainz, Germany, May 6-8, 2014. Cancer Immunol. Immunother. 64, 923-930 (2015).

17. Weber, R. et al. Myeloid-derived suppressor cells hinder the anti-cancer activity of immune checkpoint inhibitors. Front. Immunol. 9, 1310 (2018).

18. Holtzhausen, A. et al. TAM family receptor kinase inhibition reverses MDSC-mediated suppression and augments anti-PD-1 therapy in melanoma. Cancer Immunol. Res. 7, 1672-1686 (2019).

19. Villarroel-Espindola, F. et al. Spatially resolved and quantitative analysis of VISTA/PD-1H as a novel immunotherapy target in human non-small cell lung cancer. Clin. Cancer Res. 24, 1562-1573 (2018).

20. Wu, L. et al. Expression of VISTA correlated with immunosuppression and synergized with CD8 to predict survival in human oral squamous cell carcinoma. Cancer Immunol. Immunother. 66, 627-636 (2017).

21. Kuklinski, L. F. et al. VISTA expression on tumor-infiltrating inflammatory cells in primary cutaneous melanoma correlates with poor disease-specific survival. Cancer Immunol. Immunother. 67, 1113-1121 (2018).

22. Qin, S. et al. Novel immune checkpoint targets: moving beyond PD-1 and CTLA-4. Mol. Cancer 18, 155 (2019).

23. Rshenwald, J. E. et al. Melanoma staging: evidence-based changes in the American Joint Committee on Cancer eighth edition cancer staging manual. CA Cancer. J. Clin. 67, 472-492 (2017).

24. Ferris, R. L. Immunology and immunotherapy of head and neck cancer. J. Clin. Oncol. 33, 3293-3304 (2015)

25. De Costa, A. M., Schuyler, C. A., Walker, D. D. \& Young, M. R. Characterization of the evolution of immune phenotype during the development and progression of squamous cell carcinoma of the head and neck. Cancer Immunol. Immunother. 61, 927-939 (2012).

26. Gantt, S., Gervassi, A., Jaspan, H. \& Horton, H. The role of myeloid-derived suppressor cells in immune ontogeny. Front. Immunol. 5, 387 (2014).

27. Chandra, D. \& Gravekamp, C. Myeloid-derived suppressor cells: cellular missiles to target tumors. Oncoimmunology 2, e26967 (2013).

28. Brandau, S., Moses, K. \& Lang, S. The kinship of neutrophils and granulocytic myeloid-derived suppressor cells in cancer: cousins, siblings or twins?. Semin. Cancer Biol. 23, 171-182 (2013).

29. Ostrand-Rosenberg, S., Sinha, P., Beury, D. W. \& Clements, V. K. Cross-talk between myeloid-derived suppressor cells (MDSC), macrophages, and dendritic cells enhances tumor-induced immune suppression. Semin. Cancer Biol. 22, 275-281 (2012).

30. Filipazzi, P., Huber, V. \& Rivoltini, L. Phenotype, function and clinical implications of myeloid-derived suppressor cells in cancer patients. Cancer Immunol. Immunother. 61, 255-263 (2012).

31. Ostrand-Rosenberg, S. \& Sinha, P. Myeloid-derived suppressor cells: linking inflammation and cancer. J. Immunol. 182, 4499-4506 (2009).

32. Munn, D. H. \& Bronte, V. Immune suppressive mechanisms in the tumor microenvironment. Curr. Opin. Immunol. 39, 1-6 (2016).

33. Noman, M. Z. et al. PD-L1 is a novel direct target of HIF-1a, and its blockade under hypoxia enhanced MDSC-mediated T cell activation. J. Exp. Med. 211, 781-790 (2014).

34. Ridder, K. et al. Extracellular vesicle-mediated transfer of functional RNA in the tumor microenvironment. Oncoimmunology 4, e1008371 (2015)

35. Nam, S., Lee, A., Lim, J. \& Lim, J. S. Analysis of the expression and regulation of PD-1 protein on the surface of myeloid-derived suppressor cells (MDSCs). Biomol. Ther. (Seoul) 27, 63-70 (2019).

36. Boyd-Kirkup, J. et al. HMBD004, a novel anti-CD47xCD33 bispecific antibody displays potent anti-tumor effects in pre-clinical models of AML. Blood 130, 1378-1378 (2017).

37. Laszlo, G. S., Estey, E. H. \& Walter, R. B. The past and future of CD33 as therapeutic target in acute myeloid leukemia. Blood Rev. 28, 143-153 (2014).

38. Hernandez-Caselles, T. et al. A study of CD33 (SIGLEC-3) antigen expression and function on activated human T and NK cells: two isoforms of CD33 are generated by alternative splicing. J. Leukoc. Biol. 79, 46-58 (2006).

39. Dworzak, M. N., Fritsch, G., Froschl, G., Printz, D. \& Gadner, H. Four-color flow cytometric investigation of terminal deoxynucleotidyl transferase-positive lymphoid precursors in pediatric bone marrow: CD79a expression precedes CD19 in early B-cell ontogeny. Blood 92, 3203-3209 (1998).

40. Scheinberg, D. A. et al. A phase I trial of monoclonal antibody M195 in acute myelogenous leukemia: specific bone marrow targeting and internalization of radionuclide. J. Clin. Oncol. 9, 478-490 (1991).

41. Schwartz, M. A. et al. Dose-escalation trial of M195 labeled with iodine 131 for cytoreduction and marrow ablation in relapsed or refractory myeloid leukemias. J. Clin. Oncol. 11, 294-303 (1993).

42. Jurcic, J. G. et al. Targeted alpha particle immunotherapy for myeloid leukemia. Blood 100, 1233-1239 (2002).

43. Huang, X. et al. VISTA: an immune regulatory protein checking tumor and immune cells in cancer immunotherapy. J. Hematol. Oncol. 13, 83 (2020).

44. Liu, J. et al. Immune-checkpoint proteins VISTA and PD-1 nonredundantly regulate murine T-cell responses. Proc. Natl. Acad. Sci. U. S. A. 112, 6682-6687 (2015).

45. Kakavand, H. et al. Negative immune checkpoint regulation by VISTA: a mechanism of acquired resistance to anti-PD-1 therapy in metastatic melanoma patients. Mod. Pathol. 30, 1666-1676 (2017).

\section{Acknowledgements}

The Institutional Review Board of Asan Medical Center approved this study (2017-102). This work was supported by a grant from the National Research Foundation of Korea (NRF), funded by the Ministry of Science and Information Technology (MSIT) of the Korean government (NRF-2018R1C1B6003389). 


\section{Author contributions}

J.W.C., Y.J.K., W.J.L. and S.E.C. had full access to all the data in the study and take responsibility for the integrity of the data and the accuracy of the data analysis. Concept and design, data acquisition, data analysis/interpretation, drafting manuscript, critical revision of manuscript, and final approval: J.W.C., Y.J.K., K.A.Y., C.H.W., M.W.L., J.H.C., S.E.C. and W.J.L. Statistical analysis: J.W.C., Y.J.K. and Y.J.L. Technical support: W.J.L. and S.E.C. Supervision: W.J.L. All authors reviewed the manuscript.

\section{Competing interests}

The authors declare no competing interests.

\section{Additional information}

Supplementary information is available for this paper at https://doi.org/10.1038/s41598-020-71216-2.

Correspondence and requests for materials should be addressed to S.E.C. or W.J.L.

Reprints and permissions information is available at www.nature.com/reprints.

Publisher's note Springer Nature remains neutral with regard to jurisdictional claims in published maps and institutional affiliations.

(c) (i) Open Access This article is licensed under a Creative Commons Attribution 4.0 International License, which permits use, sharing, adaptation, distribution and reproduction in any medium or format, as long as you give appropriate credit to the original author(s) and the source, provide a link to the Creative Commons license, and indicate if changes were made. The images or other third party material in this article are included in the article's Creative Commons license, unless indicated otherwise in a credit line to the material. If material is not included in the article's Creative Commons license and your intended use is not permitted by statutory regulation or exceeds the permitted use, you will need to obtain permission directly from the copyright holder. To view a copy of this license, visit http://creativecommons.org/licenses/by/4.0/.

(C) The Author(s) 2020 